\title{
The role of Sergentomyia schwetzi in epidemiology of visceral leishmaniasis in Ethiopia
}

\author{
N Polanska*, I Rohousova, P Volf \\ From The 1st Conference on Neglected Vectors and Vector-Borne Diseases (EurNegVec): with Management \\ Committee and Working Group Meetings of the COST Action TD1303 \\ Cluj-Napoca, Romania. 8-11 April 2014
}

Leishmaniasis is caused by a protozoan of the genus Leishmania and transmitted by the bites of phlebotomine sand flies. During the blood feeding, sand fly females inject saliva into the host thus affecting Leishmania transmission; in a naive host saliva enhances parasite virulence, in preexposed host it acts as the protective immunogenic agent by eliciting anti-saliva specific cellular and antibody immune response. Interestingly, anti-saliva antibodies in bitten hosts can be used in epidemiological studies as the marker of exposure and the risk marker of Leishmania transmission.

Ethiopia is endemic for visceral leishmaniasis caused by Leishmania donovani and transmitted mainly by Phlebotomus orientalis. However, the most abundant sand flies in the area belong to the genus Sergentomyia. Sergentomyia females prefer to feed on reptiles, but several studies reported mammals as the additional blood source. The main aim of this study was to determine, whether $S$. schwetzi frequently bite domestic animals and thus may play some role in the pathogen transmission.

Sera of domestic animals collected in three leishmaniasis foci were tested for anti-S. schwetzi IgG antibodies by ELISA using $S$. schwetzi salivary gland homogenate as an antigen. Altogether we tested 603 serum samples from five species: cattle, sheep, goats, donkeys and dogs. Sera of animals from nonendemic countries served as a negative control and the results were statistically evaluated.

Significant seropositivity for anti-S. schwetzi IgG was found in about one third of domestic animals tested. The highest seropositivity was found in sheep (115/181), cattle $(25 / 108)$ and goats $(26 / 144)$, followed by donkeys $(2 / 24)$ and dogs (10/37).

\footnotetext{
* Correspondence: polanskn@natur.cuni.cz Department of Parasitology, Faculty of Science, Charles University in Prague, Prague, Czech Republic
}

Cite this article as: Polanska et al:: The role of Sergentomyia schwetzi in epidemiology of visceral leishmaniasis in Ethiopia. Parasites \& Vectors 2014 7(Suppl 1):05. and take full advantage of:

- Convenient online submission

- Thorough peer review

- No space constraints or color figure charges

- Immediate publication on acceptance

- Inclusion in PubMed, CAS, Scopus and Google Scholar

- Research which is freely available for redistribution

Our results suggest that sand flies of the genus Sergentomyia frequently bite domestic animals in Ethiopia. However, further studies are needed to investigate the role of Sergentomyia in transmission cycle of veterinary important pathogens, including Leishmania sp.

collowing grant projects: 13-05292S and Bill and Melinda Published: 1 April 2014 